\title{
Reggeon field theory RFT as an effective theory for QCD in Regge Limit
}

\section{Carlos Contreras*}

Departamento de Fisica, Univ. Tecnica Federico Santa Maria, Valparaiso - Chile

E-mail: carlos.contreraseusm.cl

\section{Jochen Bartels}

Affiliation Hamburg University

E-mail: jochen.bartels@desy.de

\section{Gian Paolo Vacca}

Affiliation INFA Bologna Italy

E-mail: vacca@bo.infn.it

In this work, we will discuss if the Reggeon field theory might serve as an effective theory of high energy scattering for strong interaction. Using the Functional Renormalization approach we will present results about the critical properties of the RFT: Flow equation, fixed points conditions, critical exponents evaluation and discuss the effect of the truncation on this analysis. Finally I will discuses the anomalous dimension effect and physical applications.

38th International Conference on High Energy Physics

3-10 August 2016

Chicago, USA

\footnotetext{
* Speaker.

${ }^{\dagger}$ This work has been supported by Grants Fondecyt: 1120360, 1140842 and by DGIP/USM 2016.
} 


\section{Introduction}

To find a bridge between short and long distances physics in the high energy QCD remains a topic of high interest. At small transverse distances where perturbation theory can be applied QCD predicts the BFKL Pomeron ( [1] and refs. therein) and more general, the BFKL Pomeron can be seen as the basic building block of QCD reggeon field theory in which the reggeized gluon is the basic field and the Pomeron is generated as a composite state of two or more reggeized gluons. Within this perturbative QCD reggeon field theory, the BFKL Pomeron is expected to receive corrections, have self interactions leading to Pomeron loops etc. In this case one can calculate the hard BFKL Pomeron Intercept: $\alpha_{P}=1+\mu=1.4$. On the other hand, high energy scattering of hadrons strongly depends upon large transverse distances where perturbation theory is not applicable. As the most promising theoretical concept, again Regge theory (Regge trajectory $\alpha\left(t=-q^{2}\right)=\alpha(0)-\alpha^{\prime} q^{2}$, and $\alpha^{\prime}$ Pomeron slope) can be used, where the total cross section is given by $\sigma=A_{0} s^{\alpha(0)-1}$ and the parameters have to be taken from data. Most prominent and phenomenologically successful examples include the Regge pole model of Donnachie and Landshoff [2], where from Fig. 2b. the $\mu_{p p}=\alpha(0)-1=0.08$. We therefore see strong evidence that in both regions - short and long transverse distances - we have the same structure: reggeon field (RFT) which lives in one time (rapidity) and two space dimensions (transverse distances)

\section{Functional Renormalization Group FRG}

The tool we are going to use is the FRG technique in an essentially Wilsonian form, i.e. we shall make use of the effective average action (EAA) description, which allows to study the change of the generator of the proper vertices of the theory as one integrates over the ultraviolet field modes. The analysis of the flow equation for the effective average action has successfully been applied to numerous problems in statistical mechanics, in particle physics, and in quantum gravity. We can write down the Exact FRG (Wetterich [3]) equation for $\Gamma_{k}$ :

$$
\partial_{t} \Gamma_{k}=\frac{1}{2} \operatorname{Tr}\left[\Gamma_{k}^{(2)}+\mathbb{R}_{k}\right]^{-1} \partial_{t} \mathbb{R}_{k} .
$$

For constant fields the propagator on the rhs of the flow equation (2.1) is derived from:

$$
\Gamma_{k}^{(2)}+\mathbb{R}_{k}=\left(\begin{array}{cc}
V_{k \psi \psi} & -i Z_{k} \omega+Z_{k} \alpha_{k}^{\prime} q^{2}+R_{k}+V_{k \psi \psi^{\dagger}} \\
i Z_{k} \omega+Z_{k} \alpha_{k}^{\prime} q^{2}+R_{k}+V_{k \psi^{\dagger} \psi} & V_{k \psi^{\dagger} \psi^{\dagger}}
\end{array}\right) .
$$

where $\mathbb{R}_{k}$ is the cut-off regulator and inserting the inverse of this into the rhs of (2.1) we arrive at a partial differential equation for the potential $V_{k}$ which provides the starting point of our analysis.

\subsection{Flow equations for the Reggeon Field Theory}

Let us now consider the RFT, in the lowest truncation of the local field approximation, where the effective action is a function of the pomeron field $\psi$ and its hermitian conjugate $\psi^{\dagger}$, and it is:

$$
\Gamma_{k}\left[\psi^{\dagger}, \psi\right]=\int \mathrm{d}^{D} x \mathrm{~d} \tau\left(Z_{k}\left(\frac{1}{2} \psi^{\dagger} \partial_{\tau}^{\leftrightarrow} \psi-\alpha_{k}^{\prime} \psi^{\dagger} \nabla^{2} \psi\right)+V_{k}\left[\psi^{\dagger}, \psi\right]\right)
$$


where $\tau$ is the rapidity, the dimension $D=2$ of the transverse space and the potential $V_{k}$ has the general properties: it is symmetric under the interchange $\psi \leftrightarrow \psi^{\dagger}$, and for real values of $\psi$ and $\psi^{\dagger}$, the real part of $V_{k}$ is symmetric under $\psi \rightarrow-\psi, \psi^{\dagger} \rightarrow-\psi^{\dagger}$, the imaginary part odd. In a polynomial expansion in the region of small fields, this implies that even powers of the field variables come with real coefficients (couplings), whereas odd powers have imaginary coefficients. This, in particular, ensures the negative sign of closed Pomeron loops.

\section{Search for fixed points and anomalous dimensions (LPA')}

Generically we write the truncation of the potential in terms of $\psi, \psi^{\dagger}$, or we may use: $r=$ $\psi^{\dagger} \psi, u=\psi+\psi^{\dagger}$ and with these variables, we can write a completely equivalent expansion around the origin;

$$
V_{k}=\sum_{n, m} V_{n m}(k)\left(\psi-\psi_{0}\right)^{n}\left(\psi^{\dagger}-\psi_{0}^{\dagger}\right)^{m} \text { or } V_{k}=\sum_{n, m \geq 0} \lambda_{n, m}(k) r^{1+n}(i u)^{m}
$$

with real-valued constants $\lambda_{n, m}$. Calculating the flow equation (2.1), we can obtain the beta functions for the dimensionless running couplings $\lambda_{n, m}(k)$ of the RFT. To move to dimensionless quantities we shall use: $[x]=k^{-1},[\tau]=E^{-1}$ so that $[\psi]=\left[\psi^{\dagger}\right]=k^{D / 2},\left[\alpha^{\prime}\right]=E k^{-2}$ and $\tilde{\psi}=$ $Z_{k}^{\frac{1}{2}} k^{-D / 2} \psi, \tilde{V}_{k}=\frac{V_{k}}{\alpha_{k}^{\prime} k^{D+2}}$. In Fig.1a we show the non trivial fixed point at $\left(\mu^{*}=0.11, \lambda^{*}=1.05\right)$, considering a truncation of $V_{k}$ up to cubic order and with cut-off regulator $R_{k}(q)=Z_{k} \alpha_{k}^{\prime}\left(k^{2}-\right.$ $\left.q^{2}\right) \Theta\left(k^{2}-q^{2}\right):$
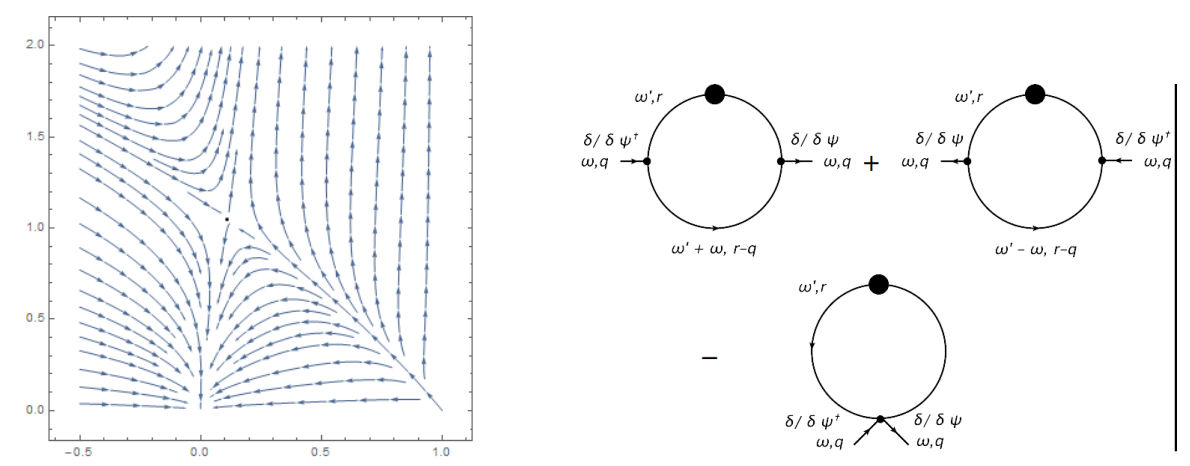

Figure 1: (a) Flow portrait $\mu, \lambda$ for potential up to cubic order. (b) Loop expansion to calculate the anomalous dimensions

In Table 1 we list, for different truncations, the fixed points and the critical exponent $v$ which is defined as the negative inverse of the negative eigenvalue:

\begin{tabular}{|l|c|c|c|c|c|c|c|c|c|c|}
\hline truncation & 3 & 4 & 5 & 6 & 7 & 8 & 9 & 10 & 11 & 12 \\
\hline exponent $v$ & 0.53 & 0.59 & 0.59 & 0.78 & 0.76 & 0.72 & 0.72 & 0.74 & 0.74 & 0.73 \\
\hline mass $\tilde{\mu}$ & 0.111 & 0.274 & 0.386 & 0.429 & 0.341 & 0.388 & 0.388 & 0.400 & 0.399 & 0.397 \\
\hline$i \psi_{0, \text { diag }}$ & 0.035 & 0.059 & 0.072 & 0.078 & 0.075 & 0.073 & 0.073 & 0.074 & 0.074 & 0.074 \\
\hline$i \psi_{0, \text { axis }}$ & 0.106 & 0.175 & 0.214 & 0.228 & 0.221 & 0.215 & 0.217 & 0.219 & 0.219 & 0.218 \\
\hline
\end{tabular}

Table 1: Parameters at the fixed point for different truncations around $\left(\psi, \psi^{\dagger}\right)=(0,0)$. 
For comparison, the critical value obtained from a Monte Carlo simulation is $v=0.73^{1}$.

Numerical results for $(r, u)=\left(0, u_{0}\right)$ are shown in Table 2 and the sequence of truncations shows a good convergence:

\begin{tabular}{|l|c|c|c|c|c|c|}
\hline truncation & 3 & 4 & 5 & 6 & 7 & 8 \\
\hline exponent $v$ & 0.74 & 0.75 & 0.73 & 0.73 & 0.73 & 0.73 \\
\hline mass $\tilde{\mu}_{\text {eff }}$ & 0.33 & 0.362 & 0.384 & 0.383 & 0.397 & 0.397 \\
\hline$i \psi_{0, \text { diag }}$ & 0.058 & 0.072 & 0.074 & 0.074 & 0.0 .074 & 0.074 \\
\hline$i u_{0}$ & 0.173 & 0.213 & 0.218 & 0.218 & 0.218 & 0.218 \\
\hline
\end{tabular}

Table 2: Parameters of the fixed point for different truncations around $(r, u)=\left(0, u_{0}\right)$.

Results with anomalous dimensions $\eta_{k}=-\frac{1}{Z_{k}} \partial_{t} Z_{k}$ and $\zeta_{k}=-\frac{1}{\alpha_{k}^{\prime}} \partial_{t} \alpha_{k}^{\prime}$ are shown in Table 3: the sequence of truncations shows a good convergence, and the numerical values of $\eta^{*}, \zeta *$ are small:

\begin{tabular}{|l|c|c|c|c|c|c|}
\hline truncation & 3 & 4 & 5 & 6 & 7 & 8 \\
\hline exponent $v$ & 0.660 & 0.659 & 0.635 & 0.633 & 0.634 & 0.634 \\
\hline mass $\tilde{\mu}_{e f f}$ & 0.356 & 0.376 & 0.396 & 0.396 & 0.414 & 0.414 \\
\hline anom.dim. $\eta$ & -0.054 & -0.087 & -0.081 & -0.080 & -0.080 & -0.080 \\
\hline anom.dim. $\zeta$ & 0.061 & 0.113 & 0.118 & 0.117 & 0.117 & 0.117 \\
\hline$i \psi_{0, \text { diag }}$ & 0.0593 & 0.0754 & 0.0766 & 0.0763 & 0.0762 & 0.0763 \\
\hline$i u_{0}$ & 0.178 & 0.222 & 0.226 & 0.225 & 0.225 & 0.225 \\
\hline
\end{tabular}

Table 3: Polynomial expansion around $(r, u)=\left(0, u_{0}\right)$ with anomalous dimensions.

A comparison with Table 2 shows, for the parameters of the potential, a strong similarity: the effective mass $\mu$ ( 0.414 vs. 0.397$)$, the position of the stationary point on the diagonal ( $0.0763 \mathrm{vs}$ $0.074)$ and on the axis $(0.225$ vs 0.218$)$. The quantity which changes more is the critical exponent $v$, which seems to become worse, if we compare it to numerical Monte Carlo estimates, which where very close to the asymptotic values. The reason for this should be found in the poorness of the anomalous dimension estimates, which strongly depends here on the field configuration chosen.

Finally, in Table 4 we summarize and compare our results in $D=2$ spatial dimensions, obtained in LPA's, with the two-loop $\varepsilon$-expansion and with recent Monte Carlo data:

\begin{tabular}{|c|c|c|c|c|c|}
\hline & LPA & LPA $^{\prime}$ & LPA' $_{2}$ & 2-loop $\varepsilon$-exp. & MC \\
\hline$v$ & 0.730 & 0.634 & 0.771 & 0.709 & 0.729 \\
\hline$z$ & 2 & 1.883 & 1.911 & 1.716 & 1.766 \\
\hline$\beta$ & 0.730 & 0.608 & 0.669 & 0.622 & 0.580 \\
\hline
\end{tabular}

Table 4: Comparison of our results with the $\varepsilon$ expansion and MC results for percolation in

$$
d=2+1 \text {. }
$$

\footnotetext{
${ }^{1}$ It has been shown long ago by J. Cardy [4] that RFT belongs to the same universality class as the critical phenomena known as directed percolation.
} 
One notices that the determination of the critical exponent $v$ is almost exact in the simplest LPA truncation. This is a common feature in several FRG studies applied to other theories. In contrast, $\beta$ is better approximated in the LPA' truncation. We notice that when taking into account the anomalous dimension in LPA' scheme, this truncation reduces the quality of the estimate for $v$.

Finally, our discussion has been formulated in terms of dimensionless parameters. Physical quantities, i.e. the value of the Pomeron intercept, carry dimension, is showed in the Fig. 2a., where $t=\ln k / k_{0}$ :
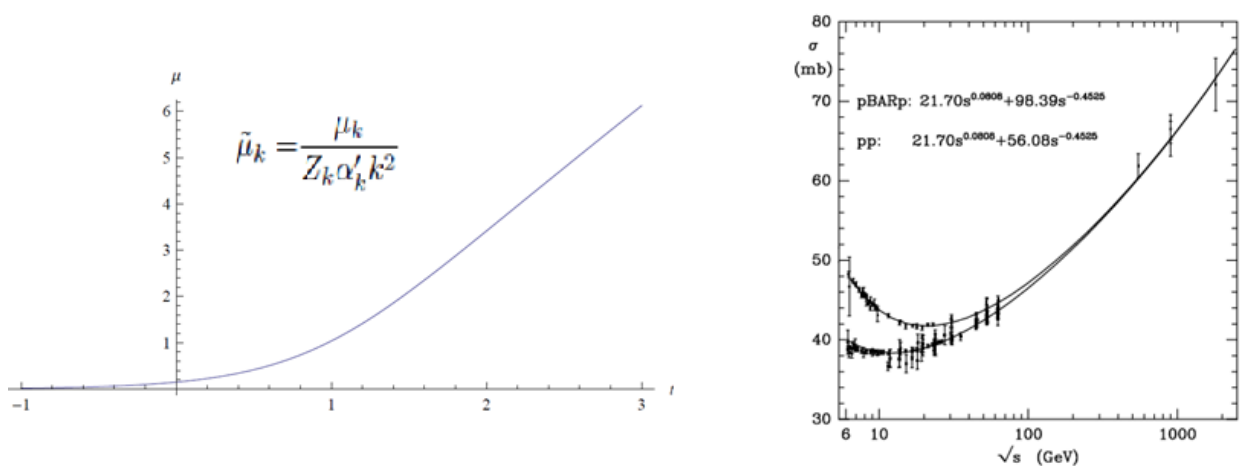

Figure 2: (a) Running Pomeron intercept $\mu_{k}=\alpha_{k}-1$. (b) Total cross section pp-Scattering.

From this figure we can see the behavior of the running Pomeron intercept in the UV region.

\section{Conclusions}

As our main result we have found that there exist a non trivial interacting fixed point (for dimensionless quantities) with one single relevant direction associated to it. Moreover, the $\mu$-value associated with the fixed point is positive, i.e. the corresponding intercept is greater than one and in the UV region (short distance region) the BFKL Pomeron predicts an intercept greater than one.

\section{References}

[1] J. Bartels, C. Contreras and G. P. Vacca: Could Reggeon field theory be and effective theory for QCD in the Regge limit, JHEP $\mathbf{1 6 0 3}$ (2016) 201 [arXiv:1512.07182].

[2] A. Donnachie and P.V. Landshoff: pp total cross section and elastic scattering, Phys. Lett. B 727 (2013) 500 [arXiv:1309.1292]

[3] C. Wetterich: Exact Evolution equation for the effective potential, Phys. Lett. B 329 (1994) 241 [arXiv: 940.3340$]$

[4] J. Cardy and L. Sugar: Directed Percolation and Reggeon Field Theory, J. Phys. A 13 (1980) 423 [hep-th/1512.07182] 